\title{
A Concise Asymmetric Route to the Bridged Bicyclic Tropane Alkaloid Ferruginine using Enyne Ring Closing Metathesis
}

\author{
Varinder K. Aggarwal, ${ }^{*}$ Christopher J. Astle and Mark Rogers-Evans ${ }^{\dagger}$ \\ School of Chemistry, Bristol University, Cantock's Close, Bristol BS8 1TS, UK.
}

v.aggarwal@bristol.ac.uk

* Corresponding Author

† F. Hoffmann-La Roche Ltd, Pharmaceuticals Division

Lead Generation, PRBC-CI, Bldg 65/416

CH-4070 Basel (Switzerland)

\section{Supporting Information}

Infrared spectra were recorded on a Perkin-Elmer 157G FT-IR. Samples were recorded as a thin film.

${ }^{1} \mathrm{H}$ NMR spectra were recorded at $400 \mathrm{MHz}$ using a Jeol Delta GX instrument.

${ }^{13} \mathrm{C}$ NMR spectra were recorded at $100 \mathrm{MHz}$ on a GX400 spectrometer.

Low-resolution electron impact mass spectra $(\mathrm{m} / \mathrm{z})$ were recorded with a Fisons/VG Autospec System.

Elemental analyses were determined on a Perkin-Elmer 2400 CHN microanalyser.

Analytical TLC was performed on Merck Kieselgel $60 \mathrm{~F}_{254}$ aluminium.

Melting points were determined on a Kholfer hotstage.

The following reagents were used as obtained: L-pyroglutamic acid was obtained from Aldrich; Dess-Martin periodinane was obtained from Lancaster; Grubbs $1^{\text {st }}$ generation catalyst I was obtained from Fluka; Grubbs $2^{\text {nd }}$ generation catalyst II was obtained from Strem Chemicals. $\mathrm{CH}_{3} \mathrm{COCN}_{2} \mathrm{PO}(\mathrm{OEt})_{2}$ was prepared as described in the literature. ${ }^{[1]}$

Reactions requiring anhydrous conditions were performed in vacuum heat-dried glassware under nitrogen atmospheres. Reaction mixtures were stirred magnetically. The dry solvents $\mathrm{MeCN}$, benzene, toluene, and $\mathrm{MeOH}$ were obtained from a sure seal bottle. DCM, THF, $\mathrm{Et}_{2} \mathrm{O}$ were obtained from the dry solvent dispenser at the University of Bristol which is built by Anhydrous Engineering and is based on the Grubbs design. ${ }^{[2]}$ 


\section{2-Benzyl 1-tert-butyl (2S)-5-oxo-1,2-pyrrolidinedicarboxylate $(5)^{[3]}$}

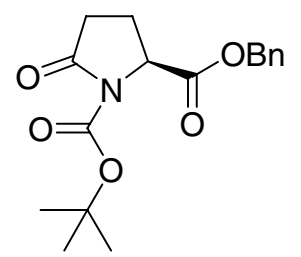

5

To a stirred solution of L-pyroglutamic acid (24 g, $186 \mathrm{mmol})$ and $N, N$ diisopropylethylamine $(38.8 \mathrm{ml}, 223 \mathrm{mmol})$ in $\mathrm{CH}_{2} \mathrm{Cl}_{2}(500 \mathrm{ml})$ at $0{ }^{\circ} \mathrm{C}$ was added benzyl bromide $(22.1 \mathrm{ml}, 186 \mathrm{mmol})$, the mixture was heated at reflux for 5 hours, cooled to r.t., and washed with aqueous $\mathrm{NaH}_{2} \mathrm{PO}_{4(\mathrm{aq})}(2 \times 50 \mathrm{ml}, 0.2 \mathrm{M})$. The aqueous layer was extracted with $\mathrm{CH}_{2} \mathrm{Cl}_{2}(3 \times 50 \mathrm{ml})$, and the combined organic layers were washed with brine $(25 \mathrm{ml})$, dried over $\mathrm{MgSO}_{4}$, filtered and concentrated in vacuo. The crude product was then dissolved in a solution of $\mathrm{CH}_{3} \mathrm{CN}(500 \mathrm{ml})$ containing 4dimethylaminopyridine $(2.27 \mathrm{~g}, 18.6 \mathrm{mmol})$ and di-tert-butyl dicarbonate $(48.8 \mathrm{~g}, 223$ mmol), and stirred at r.t. for 3 hours. The yellow mixture was washed with $\mathrm{NaH}_{2} \mathrm{PO}_{4(\mathrm{aq})}(2 \times 50 \mathrm{ml}, 0.2 \mathrm{M})$ and brine $(25 \mathrm{ml})$, dried over $\mathrm{MgSO}_{4}$, filtered, and concentrated in vacuo to give a coloured viscous solid. Purification by column chromatography eluting with $40 \%$ EtOAc in pet. ether, yielded $5(37.5 \mathrm{~g}, 92 \%)$ as a colourless crystalline solid;

TLC $\mathrm{R}_{f} 0.62$ (20\% EtOAc in DCM); m.p. $72-73{ }^{\circ} \mathrm{C}$ \{lit. 69-70 ${ }^{\circ} \mathrm{C},{ }^{[3]} 57-59{ }^{\circ} \mathrm{C},{ }^{[4]} 72-74$ $\left.{ }^{\circ} \mathrm{C}^{[5]}\right\} ;[\alpha]^{20}{ }_{\mathrm{D}}-34.2\left(c 0.3, \mathrm{CHCl}_{3}\right)$ \{lit. $-37.8\left(\right.$ c 1.0, $\left.\mathrm{CHCl}_{3}\right),{ }^{[3]}-35.0\left(\right.$ c $\left.0.98, \mathrm{CHCl}_{3}\right)$

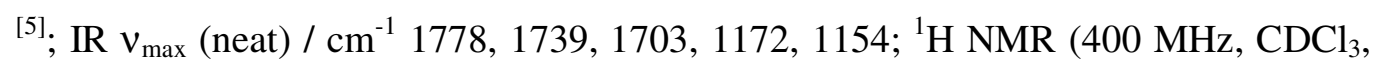
$\left.25^{\circ} \mathrm{C}, \mathrm{TMS}\right) \delta=7.39-7.33(5 \mathrm{H}, \mathrm{m}, \mathrm{Ph}), 5.23\left(1 \mathrm{H}, \mathrm{d}, J 12.2 \mathrm{~Hz}, \mathrm{CH}_{\mathrm{A}} \mathrm{CH}_{\mathrm{B}} \mathrm{Ph}\right), 5.19(1$ $\left.\mathrm{H}, \mathrm{d}, J 12.2 \mathrm{~Hz}, \mathrm{CH}_{\mathrm{A}} \mathrm{CH}_{\mathrm{B}} \mathrm{Ph}\right), 4.65(1 \mathrm{H}, \mathrm{dd}, J 9.5$ and $2.6 \mathrm{~Hz}, \mathrm{NCH}), 2.66-1.98(4 \mathrm{H}$, $\left.\mathrm{m}, \mathrm{CH}_{2} \mathrm{CH}_{2}\right), 1.42\left(9 \mathrm{H}, \mathrm{s}, \mathrm{OCMe}_{3}\right) ;{ }^{13} \mathrm{C} \mathrm{NMR}\left(100 \mathrm{MHz}, \mathrm{CDCl}_{3}, 25{ }^{\circ} \mathrm{C}\right) \delta=173.2$, 171.2, 149.3, 135.1, 128.6, 128.7, 128.8, 83.6, 67.4, 59.0, 31.2, 27.9, 21.6; MS m/z (EI) $84\left(\mathrm{M}^{+}-\mathrm{CO}_{2} \mathrm{Bn}-\mathrm{BOC}, 100 \%\right), 77$ (10\%), 65 (15\%). 
Preparation of compound 6

2-Benzyl 1-tert-butyl (2S,5S)-5-hydroxy-1,2-pyrrolidinedicarboxylate (cis - 17) and 2-benzyl 1-tert-butyl $(2 S, 5 R)$-5-hydroxy-1,2-pyrrolidinedicarboxylate (trans 17)

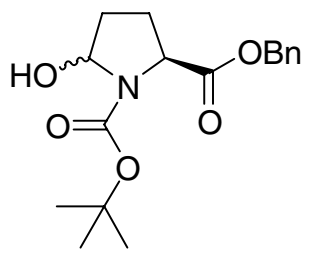

17

To a stirred solution of $5(16.6 \mathrm{~g}, 52.2 \mathrm{mmol})$ in $\mathrm{THF}(25 \mathrm{ml})$ at $-78{ }^{\circ} \mathrm{C}$ under nitrogen, was added lithiumtriethyl borohydride (62.6 ml, $1.0 \mathrm{M}$ in THF, $62.6 \mathrm{mmol})$ dropwise over 5 minutes. After 2 hours, the reaction was quenched by the addition of a saturated solution of $\mathrm{NaHCO}_{3(\mathrm{aq})}(10 \mathrm{ml})$, followed by the addition of $\mathrm{Et}_{2} \mathrm{O}(20 \mathrm{ml})$. The aqueous layer was extracted with $\mathrm{Et}_{2} \mathrm{O}(3 \times 30 \mathrm{ml})$ and the combined organics washed with brine $(30 \mathrm{ml})$, dried over $\mathrm{MgSO}_{4}$, filtered and concentrated in vacuo to yield light yellow oil. Purification by column chromatography eluting with $20 \%$ EtOAc in pet. ether, yielded $17(14.8 \mathrm{~g}, 88 \%)$ as a clear gum which gave a single peak by GCMS, but was clearly a mixture of two diastereoisomers by ${ }^{13} \mathrm{C}$ and ${ }^{1} \mathrm{H}$ NMR measured at $100{ }^{\circ} \mathrm{C}$ (to eliminate rotomer isomers) in a ratio of 75:25;

TLC $\mathrm{R}_{f} 0.42$ (40\% EtOAc in DCM); IR $v_{\max }$ (neat) $/ \mathrm{cm}^{-1} 3420,1745,1688,1366$, 1156; ${ }^{1} \mathrm{H}$ NMR (400 MHz, $d_{6}$-DMSO, $\left.100{ }^{\circ} \mathrm{C}\right) \delta=7.39-7.34(5 \mathrm{H}, \mathrm{m}, \mathrm{Ph}), 5.45-5.41$ (1 H, m, CHOH) 5.21-5.15 (2 H, m, $\left.\mathrm{CH}_{2} \mathrm{Ar}\right), 4.42\left(0.75 \mathrm{H}, \mathrm{d}, J 9.0 \mathrm{~Hz}, \mathrm{NCHCO}_{2} \mathrm{Bn}\right)$, $4.23\left(0.25 \mathrm{H}, \mathrm{t}, J\right.$ 8.0 Hz, $\left.\mathrm{NCHCO}_{2} \mathrm{Bn}\right), 2.49-2.40\left(1 \mathrm{H}, \mathrm{m}, \mathrm{CH}_{\mathrm{A}} \mathrm{CH}_{\mathrm{B}} \mathrm{CH}_{2}\right), 2.28-2.05$ $\left(1 \mathrm{H}, \mathrm{m}, \mathrm{CH}_{\mathrm{A}} \mathrm{CH}_{\mathrm{B}} \mathrm{CH}_{2}\right), 1.99-1.80\left(1 \mathrm{H}, \mathrm{m}, \mathrm{CH}_{2} \mathrm{CH}_{\mathrm{A}} \mathrm{CH}_{\mathrm{B}}\right), 1.79-1.65(1 \mathrm{H}, \mathrm{m}$, $\left.\mathrm{CH}_{2} \mathrm{CH}_{\mathrm{A}} \mathrm{CH}_{\mathrm{B}}\right), 1.42-1.38\left(9 \mathrm{H}, \mathrm{m}, \mathrm{OCMe}_{3}\right) ;{ }^{13} \mathrm{C} \mathrm{NMR}\left(100 \mathrm{MHz}, \mathrm{CDCl}_{3}, 2{ }^{\circ} \mathrm{C}\right.$, observed peaks) $\delta=172.4,172.4,154.2,154.2,135.5,135.5,128.7,128.6,128.6$, $128.5,128.4,128.4,82.4,82.3,81.1,81.0,66.9,66.8,59.5,59.3,31.0,31.0,28.1$, 28.1, 28.0, 27.8; MS m/z (CI) $322\left(\mathrm{MH}^{+}, 13 \%\right) 304\left(\mathrm{M}^{+}-\mathrm{OH}, 5 \%\right), 204\left(\mathrm{M}^{+}-\mathrm{OH}-\right.$ BOC, 15\%), 91 (100\%); HRMS Found: $m / z$ (CI) $204.1024 \mathrm{C}_{12} \mathrm{H}_{14} \mathrm{O}_{2} \mathrm{~N}$ requires 204.1024; Anal. Calcd for $\mathrm{C}_{17} \mathrm{H}_{23} \mathrm{NO}_{5}$ : C, 63.54; H, 7.21; N, 4.36. Found: C, 63.71; $\mathrm{H}, 7.51 ; \mathrm{N}, 4.45$. 
2-Benzyl 1-tert-butyl (2S,5S)-5-methoxy-1,2-pyrrolidinedicarboxylate (cis - 6) and 2-benzyl 1-tert-butyl (2S,5R)-5-methoxy-1,2-pyrrolidinedicarboxylate (trans - 6)

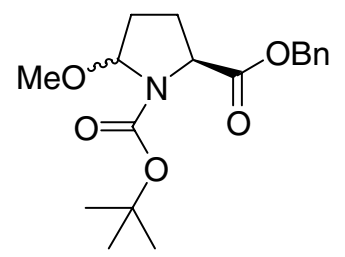

6

To a stirred solution of $\mathbf{1 7}$ (1.36 g, $4.24 \mathrm{mmol})$ in $\mathrm{MeOH}$ (25 ml) under nitrogen, was added $p$-toluenesulfonic acid $(72 \mathrm{mg}, 0.424 \mathrm{mmol})$. After 5 hours the reaction was quenched with saturated $\mathrm{NaHCO}_{3(\mathrm{aq})}(10 \mathrm{ml})$ and then concentrated in vacuo to $c a .10$ $\mathrm{ml}$, followed by the addition of $\mathrm{Et}_{2} \mathrm{O}(25 \mathrm{ml})$ and water $(25 \mathrm{ml})$. The aqueous layer was extracted with $\mathrm{Et}_{2} \mathrm{O}(3 \times 20 \mathrm{ml})$, and the combined organics were dried over $\mathrm{MgSO}_{4}$, filtered and concentrated in vacuo to yield a yellow oil. Purification by column chromatography eluting with $30 \%$ EtOAc in pet. ether, yielded 6 (1.39 g, $98 \%$ ) as a clear oil which appeared to be a single diastereomer by ${ }^{1} \mathrm{H}$ NMR measured at $100{ }^{\circ} \mathrm{C}$ (to eliminate rotomer isomers) but two by ${ }^{13} \mathrm{C}$ NMR and two by GCMS;

TLC $\mathrm{R}_{f} 0.52$ (40\% EtOAc in pet. ether); IR $v_{\max }$ (neat) / $\mathrm{cm}^{-1} 1747,1701,1155,1083$; ${ }^{1} \mathrm{H}$ NMR $\left(400 \mathrm{MHz}, d_{6}\right.$-DMSO, $\left.100{ }^{\circ} \mathrm{C}\right) \delta=7.36-7.33(5 \mathrm{H}, \mathrm{m}, \mathrm{Ph}), 5.18-5.12(3 \mathrm{H}$, $\mathrm{m}, \mathrm{CH}_{2} \mathrm{Ar}$ and $\left.\mathrm{CHOMe}\right), 4.30-4.28\left(1 \mathrm{H}, \mathrm{m}, \mathrm{NCHCO}_{2} \mathrm{Bn}\right), 3.27\left(3 \mathrm{H}, \mathrm{s}, \mathrm{CHOCH}_{3}\right)$, 2.34-1.78 (4 H, m, $\left.\mathrm{CH}_{2} \mathrm{CH}_{2}\right), 1.36\left(9 \mathrm{H}, \mathrm{s}, \mathrm{OCMe}_{3}\right) ;{ }^{13} \mathrm{C} \mathrm{NMR}\left(100 \mathrm{MHz}, \mathrm{CDCl}_{3}, 25\right.$ ${ }^{\circ} \mathrm{C}$, observed peaks) $\delta=172.5,172.3,154.0,153.8,135.7,135.8,128.6,128.5,128.4$, $128.3,128.2,128.2,89.2,88.2,80.8,80.7,66.4,66.3,59.4,59.1,56.0,55.8,31.2$, 30.2, 28.2, 28.1, 27.0, 26.9; MS m/z (CI) $304\left(\mathrm{MH}^{+}-\mathrm{OMe}, 2 \%\right), 204\left(\mathrm{MH}^{+}-\mathrm{OMe}-\right.$ BOC, 10\%), 91 (100\%); HRMS Found: $m / z$ (CI) $304.1544 \mathrm{C}_{12} \mathrm{H}_{14} \mathrm{O}_{2} \mathrm{~N}$ requires 304.1548; Anal. Calcd for $\mathrm{C}_{18} \mathrm{H}_{25} \mathrm{NO}_{5}$ : C, 64.46; H, 7.51; N, 4.18. Found: C, 64.72; H, 7.83; N, 4.39. 
2-Benzyl 1-tert-butyl (2S,5R)-5-allyl-1,2-pyrrolidinedicarboxylate (cis - 7) and 2benzyl 1-tert-butyl (2S,5S)-5-allyl-1,2-pyrrolidinedicarboxylate (trans - 7)

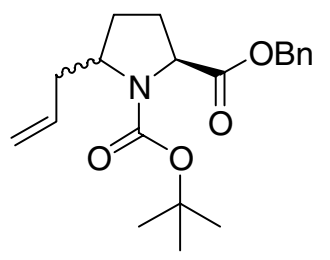

To a stirred solution of $6(203 \mathrm{mg}, 0.61 \mathrm{mmol})$ in $\mathrm{Et}_{2} \mathrm{O}(25 \mathrm{ml})$ at $-78{ }^{\circ} \mathrm{C}$ under nitrogen, was added allytrimethylsilane $(0.436 \mathrm{ml}, 2.75 \mathrm{mmol})$, followed by the dropwise addition of $\mathrm{BF}_{3} . \mathrm{Et}_{2} \mathrm{O}(77 \mu 1,0.63 \mathrm{mmol})$ over 20 minutes. After 2 hours the reaction mixture was allowed to warm to r.t. and left for a further 13 hours. $\mathrm{Et}_{2} \mathrm{O}$ (15 $\mathrm{ml})$ and water $(20 \mathrm{ml})$ was then added, the phases were separated, with the aqueous layer being extracted with $\mathrm{Et}_{2} \mathrm{O}(3 \times 20 \mathrm{ml})$ and the combined organics dried over $\mathrm{MgSO}_{4}$, filtered and concentrated in vacuo to yield a light yellow oil. Purification by column chromatography eluting with 30\% EtOAc in pet. ether yielded 7 (196 mg, $93 \%$ ) as a clear oil which gave two peaks by GCMS, and a mixture of two diastereoisomers by ${ }^{13} \mathrm{C}$ and ${ }^{1} \mathrm{H}$ NMR measured at $100{ }^{\circ} \mathrm{C}$ (to eliminate rotomer isomers) in a ratio of $8: 2$;

TLC $\mathrm{R}_{f} 0.6$ (30\% EtOAc in pet. ether); IR $v_{\max }$ (neat) $/ \mathrm{cm}^{-1} 1747,1694,1641 ;{ }^{1} \mathrm{H}$ NMR $\left(400 \mathrm{MHz}, d_{6}\right.$-DMSO, $\left.100{ }^{\circ} \mathrm{C}\right) \delta=7.28-7.23(5 \mathrm{H}, \mathrm{m}, \mathrm{Ph}), 5.76-5.63(1 \mathrm{H}, \mathrm{m}$, $\left.\mathrm{CH}_{2}=\mathrm{CHCH}_{2}\right), 5.09-4.89\left(4 \mathrm{H}, \mathrm{m}, \mathrm{CH}_{2} \mathrm{Ph}\right.$ and $\left.\mathrm{CH}_{2} \mathrm{CH}=\mathrm{CH}_{2}\right), 4.21-4.16(1 \mathrm{H}, \mathrm{m}$, $\left.\mathrm{NCHCO}_{2} \mathrm{Bn}\right), 3.87-3.78\left(0.2 \mathrm{H}, \mathrm{m}, \mathrm{NCHCH}_{2} \mathrm{CH}=\mathrm{CH}_{2}\right), 3.75(0.8 \mathrm{H}$, ddt, $J$ 7.4, 3.7 and $\left.3.4 \mathrm{~Hz}, \mathrm{NCHCH}_{2} \mathrm{CH}=\mathrm{CH}_{2}\right), 2.50-2.40\left(1 \mathrm{H}, \mathrm{m}, \mathrm{CH}_{\mathrm{A}} \mathrm{CH}_{\mathrm{B}} \mathrm{CH}=\mathrm{CH}_{2}\right), 2.20-2.03$ (2 $\mathrm{H}, \mathrm{m}, \mathrm{CH}_{\mathrm{A}} \mathrm{CH}_{\mathrm{B}} \mathrm{CH}=\mathrm{CH}_{2}$ and $\left.\mathrm{CH}_{\mathrm{A}} \mathrm{CH}_{\mathrm{B}} \mathrm{CH}_{2}\right), 1.92-1.72$ and 1.66-1.56 $(1 \mathrm{H}$ and $2 \mathrm{H}$, $\mathrm{m}, \mathrm{CH}_{\mathrm{A}} \mathrm{CH}_{\mathrm{B}} \mathrm{CH}_{2}$ ), $1.27\left(9 \mathrm{H}, \mathrm{s}, \mathrm{OCMe}_{3}\right) ;{ }^{13} \mathrm{C} \mathrm{NMR}\left(100 \mathrm{MHz}, d_{6}\right.$-DMSO, $25{ }^{\circ} \mathrm{C}$, observed peaks) $\delta=173.0,172.7,154.4,153.7,135.9,135.8,135.4,135.1,128.6$, $128.5,128.5,128.4,128.2,128.0,117.3,116.9,80.0,79.9,66.7,66.6,60.0,59.8$, 57.6, 57.5, 40.0, 38.3, 28.5, 28.4, 28.2, 28.2, 26.9, 26.9; MS m/z (CI) 304 ( $\mathrm{M}^{+}$-allyl, 23\%) $290\left(\mathrm{MH}^{+}-{ }^{t} \mathrm{Bu}, 2.5 \%\right) 246\left(\mathrm{MH}^{+}-\mathrm{BOC}, 100 \%\right), 204\left(\mathrm{M}^{+}\right.$-allyl-BOC, 95\%); HRMS Found: $m / z(C I) 246.1490 \mathrm{C}_{15} \mathrm{H}_{20} \mathrm{O}_{2} \mathrm{~N}$ requires 246.1494; Anal. Calcd for $\mathrm{C}_{20} \mathrm{H}_{27} \mathrm{NO}_{4}$ : C, 69.54; H, 7.88; N, 4.05. Found: C, 69.75; H, 7.52; N, 4.11. 
Should any $\mathrm{N}$-BOC deprotection be observed, repeating the second part of the procedure for the preparation of compound $\mathbf{5}$ ensures a high yield.

tert-Butyl (2R,5S)-2-allyl-5-(hydroxymethyl)-1-pyrrolidinecarboxylate (18)

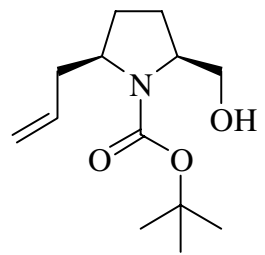

18

To a stirred solution of lithium aluminium hydride $(297 \mathrm{mg}, 7.84 \mathrm{mmol})$ in THF (5 $\mathrm{ml})$ at $0{ }^{\circ} \mathrm{C}$ was added a solution of $7(1.06 \mathrm{~g}, 3.92 \mathrm{mmol}$, an 8:2 mixture of cis:trans diastereomers) in THF $(5 \mathrm{ml})$ dropwise over 10 minutes. After 10 minutes, the reaction was warmed to r.t. and quenched by the sequential addition of water (4 ml), $15 \% \mathrm{NaOH}_{(\mathrm{aq})}$ solution $(4 \mathrm{ml})$ and then water $(12 \mathrm{ml})$, the suspension was filtered through Celite, washing with EtOAc $(10 \mathrm{ml})$. The resulting yellow solution was concentrated in vacuo to yield a yellow oil. Purification by column chromatography, eluting with $40 \%$ EtOAc in pet. ether, allowed separation of the two diastereomers, yielding pure cis - 18 (741 $\mathrm{mg}, 78 \%)$ as a clear oil;

TLC $\mathrm{R}_{f} 0.40$ (40\% EtOAc in pet. ether); $[\alpha]^{20}{ }_{\mathrm{D}}+6.3\left(c 0.3, \mathrm{CH}_{2} \mathrm{Cl}_{2}\right.$ ); IR $v_{\max }$ (neat) / $\mathrm{cm}^{-1} 3413,2974,1663 ;{ }^{1} \mathrm{H}$ NMR (400 MHz, $\mathrm{CDCl}_{3}, 25{ }^{\circ} \mathrm{C}$, TMS $) \delta=5.81-5.69(1 \mathrm{H}$, m, $\left.\mathrm{CH}_{2} \mathrm{CH}=\mathrm{CH}_{2}\right)$, 5.09-5.03 (2 H, m, $\left.\mathrm{CH}_{2} \mathrm{CH}=\mathrm{CH}_{2}\right), 4.99$ (1 H, br. s, $\left.\mathrm{OH}\right), 3.97-3.88$ $\left(2 \mathrm{H}, \mathrm{m}, \mathrm{NCHCH}_{2} \mathrm{CH}=\mathrm{CH}_{2}\right.$ and $\left.\mathrm{NCHCH}_{2} \mathrm{OH}\right), 3.70-3.63\left(1 \mathrm{H}, \mathrm{m}, \mathrm{CH}_{\mathrm{A}} \mathrm{H}_{\mathrm{B}} \mathrm{OH}\right), 3.58-$ $3.52\left(1 \mathrm{H}, \mathrm{m}, \mathrm{CH}_{\mathrm{A}} H_{\mathrm{B}} \mathrm{OH}\right), 2.50-2.45\left(1 \mathrm{H}, \mathrm{m}, \mathrm{CH}_{\mathrm{A}} \mathrm{H}_{\mathrm{B}} \mathrm{CH}=\mathrm{CH}_{2}\right), 2.28-2.18(1 \mathrm{H}, \mathrm{m}$, $\left.\mathrm{CH}_{\mathrm{A}} H_{\mathrm{B}} \mathrm{CH}=\mathrm{CH}_{2}\right), 2.02-1.98\left(1 \mathrm{H}, \mathrm{m}, \mathrm{CH}_{\mathrm{A}} \mathrm{H}_{\mathrm{B}} \mathrm{CHCH}_{2} \mathrm{OH}\right), 1.91-1.82(1 \mathrm{H}, \mathrm{m}$, $\mathrm{CH}_{\mathrm{A}} \mathrm{H}_{\mathrm{B}} \mathrm{CH}$-allyl $), 1.74-1.63\left(1 \mathrm{H}, \mathrm{m}, \mathrm{CH}_{\mathrm{A}} H_{\mathrm{B}} \mathrm{CH}\right.$-allyl $), 1.61-1.53(1 \mathrm{H}, \mathrm{m}$, $\left.\mathrm{CH}_{\mathrm{A}} \mathrm{H}_{\mathrm{B}} \mathrm{CHCH}_{2} \mathrm{OH}\right), 1.47\left(9 \mathrm{H}, \mathrm{s}, \mathrm{CMe}_{3}\right) ;{ }^{13} \mathrm{C} \mathrm{NMR}\left(100 \mathrm{MHz}, \mathrm{CDCl}_{3}, 25{ }^{\circ} \mathrm{C}\right) \delta=$ 156.4, 135.0, 117.2, 80.4, 68.1, 61.0, 58.7, 39.7, 28.5, 28.5, 26.8; MS m/z (EI) 210 $\left(\mathrm{M}^{+}-\mathrm{CH}_{2} \mathrm{OH}, 18 \%\right), 200\left(\mathrm{M}^{+}-\right.$allyl, 60\%) $154\left(\mathrm{M}^{+}-\mathrm{CH}_{2} \mathrm{OH}-{ }^{t} \mathrm{Bu}, 20 \%\right), 100\left(\mathrm{M}^{+}-\right.$ allyl-BOC, 100\%); HRMS Found: $m / z$ (EI) $210.1495 \mathrm{C}_{12} \mathrm{H}_{20} \mathrm{O}_{2} \mathrm{~N}$ requires 210.1494; Anal. Calcd for $\mathrm{C}_{13} \mathrm{H}_{23} \mathrm{NO}_{3}$ : C, 64.70; H, 9.61; N, 5.80. Found: C, 64.61; H, 9.84; N, 5.80 . 
tert-Butyl $(2 R, 5 S)$-2-allyl-5-formyl-1-pyrrolidinecarboxylate (12)

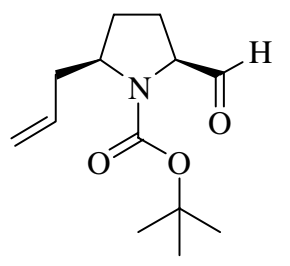

12

To a stirred solution of 18 (420 mg, $1.74 \mathrm{mmol})$ in water saturated DCM (30 ml), Dess-Martin periodinane $(1.55 \mathrm{~g}, 3.66 \mathrm{mmol})$ was added and the mixture stirred at r.t. for 30 minutes. An additional portion of water saturated DCM (4 ml) was required to drive the reaction to completion. The reaction mixture was diluted with $\mathrm{Et}_{2} \mathrm{O}(15 \mathrm{ml})$, followed by the addition of a solution of sodium thiosulfate $(3 \mathrm{~g}, 19.1 \mathrm{mmol})$ in $80 \%$ saturated solution of $\mathrm{NaHCO}_{3(\mathrm{aq})}(50 \mathrm{ml})$. The reaction mixture was stirred rapidly until the solution became clear. The aqueous layer was extracted with $\mathrm{Et}_{2} \mathrm{O}(2 \times 20$ $\mathrm{ml})$ and the combined organic layers were washed with $\mathrm{NaHCO}_{3(\mathrm{aq})}(10 \mathrm{ml})$, water (10 $\mathrm{ml})$ and brine $(10 \mathrm{ml})$, dried over $\mathrm{MgSO}_{4}$, filtered and concentrated in vacuo to provide a yellow oil. Purification by column chromatography, eluting with $40 \%$ EtOAc in pet. ether yielded pure 12 (354 mg, 85\%) as a clear oil and as a single diastereomer;

TLC $\mathrm{R}_{f} 0.51$ (40\% EtOAc in pet. ether); $[\alpha]^{20}{ }_{\mathrm{D}}-46.2\left(c 0.2, \mathrm{CH}_{2} \mathrm{Cl}_{2}\right)$; IR $v_{\max }$ (neat) / $\mathrm{cm}^{-1} 2976,1736,1686,1641 ;{ }^{1} \mathrm{H}$ NMR $\left(400 \mathrm{MHz}, d_{6}\right.$-DMSO, $\left.100{ }^{\circ} \mathrm{C}\right) \delta=9.32(1 \mathrm{H}$, s, $\mathrm{CHO}), 5.81\left(1 \mathrm{H}\right.$, ddt, $J 17,10$ and $\left.7.5 \mathrm{~Hz}, \mathrm{CH}_{2} \mathrm{CH}=\mathrm{CH}_{2}\right), 5.07(1 \mathrm{H}$, br. d, $J 17 \mathrm{~Hz}$, $\left.\mathrm{CH}_{2} \mathrm{CH}=\mathrm{CH}_{\mathrm{A}} \mathrm{H}_{\mathrm{B}}\right), 5.04\left(1 \mathrm{H}\right.$, br. d, $\left.J 10 \mathrm{~Hz}, \mathrm{CH}_{2} \mathrm{CH}=\mathrm{CH}_{\mathrm{A}} H_{\mathrm{B}}\right), 4.03-3.96(1 \mathrm{H}, \mathrm{m}$, $\mathrm{NCHCHO}), \quad 3.90-3.78$ (1 $\left.\mathrm{H}, \mathrm{m}, \quad \mathrm{NCHCH}_{2} \mathrm{CH}=\mathrm{CH}_{2}\right), \quad 2.48-2.36$ (1 $\mathrm{H}, \mathrm{m}$, $\left.\mathrm{CH}_{\mathrm{A}} \mathrm{H}_{\mathrm{B}} \mathrm{CH}=\mathrm{CH}_{2}\right), \quad 2.22-2.10 \quad\left(1 \mathrm{H}, \quad \mathrm{m}, \quad \mathrm{CH}_{\mathrm{A}} \mathrm{H}_{\mathrm{B}} \mathrm{CH}=\mathrm{CH}_{2}\right), \quad 2.00-1.80 \quad(3 \mathrm{H}, \mathrm{m}$, $\left.\mathrm{CH}_{\mathrm{A}} \mathrm{H}_{\mathrm{B}} \mathrm{CH}_{2}\right), 1.70-1.64\left(1 \mathrm{H}, \mathrm{m}, \mathrm{CH}_{\mathrm{A}} \mathrm{H}_{\mathrm{B}} \mathrm{CH}_{2}\right), 1.40\left(9 \mathrm{H}, \mathrm{s}, \mathrm{CMe}_{3}\right) ;{ }^{13} \mathrm{C}$ NMR (100 $\left.\mathrm{MHz}, \mathrm{CDCl}_{3}, 25^{\circ} \mathrm{C}\right) \delta=200.9,154.6,134.0,117.7,80.9,66.0,58.0,38.9,28.4,25.7$, 24.9; MS m/z (CI) $184\left(\mathrm{MH}^{+}-{ }^{t} \mathrm{Bu}, 95 \%\right), 154\left(\mathrm{M}^{+}-\mathrm{CHO}-{ }^{t} \mathrm{Bu}, 100 \%\right)$; HRMS Found: $m / z(C I) 184.0972 \mathrm{C}_{9} \mathrm{H}_{14} \mathrm{O}_{3} \mathrm{~N}$ requires 184.0974. 


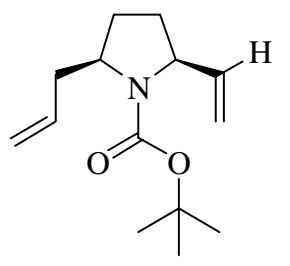

10

To a stirred slurry of methyl triphenylphosphonium bromide (460 mg, $1.29 \mathrm{mmol}$ ) in THF $(2.5 \mathrm{ml})$ at r.t. was added dropwise $n$-BuLi $(0.86 \mathrm{ml}, 1.5 \mathrm{M}$ solution in hexanes, $1.29 \mathrm{mmol}$ ) resulting in a bright yellow solution. After two hours, a solution of $\mathbf{1 2}$ (154 $\mathrm{mg}, 0.64 \mathrm{mmol})$ in THF (2 ml) was added and the reaction mixture was stirred for a further 14 hours. EtOAc $(10 \mathrm{ml})$ was added, the solution was washed with water $(10 \mathrm{ml})$, brine $(10 \mathrm{ml})$, dried over $\mathrm{MgSO}_{4}$, filtered and concentrated in vacuo to provide a yellow oil. Purification by column chromatography, eluting with $20 \%$ EtOAc in pet. ether yielded pure 10 (142 $\mathrm{mg}, 93 \%)$ as a clear oil and as a single diastereomer;

TLC $\mathrm{R}_{f} 0.57$ (20\% EtOAc in pet. ether); $[\alpha]^{20}{ }_{\mathrm{D}}+40.7\left(c 0.2, \mathrm{CH}_{2} \mathrm{Cl}_{2}\right)$; IR $v_{\max }$ (neat) / $\mathrm{cm}^{-1} 2974,2341,1689,1641 ;{ }^{1} \mathrm{H}$ NMR (400 MHz, $\left.\mathrm{CDCl}_{3}, 25^{\circ} \mathrm{C}, \mathrm{TMS}\right) \delta=5.82-5.71$ (2 $\mathrm{H}, \mathrm{m}, \mathrm{CH}_{2} \mathrm{CH}=\mathrm{CH}_{2}$ and $\mathrm{CH}_{2}=\mathrm{CHCHN}$ ), 5.15-5.01 (4 H, m, $\mathrm{CH}_{2} \mathrm{CH}=\mathrm{CH}_{2}$ and $\left.\mathrm{CH}_{2}=\mathrm{CHCH}\right), 4.27\left(1 \mathrm{H}\right.$, unresolved br. s, $\left.\mathrm{NCHCH}=\mathrm{CH}_{2}\right), 3.85(1 \mathrm{H}$, unresolved br. s, $\left.\mathrm{NCHCH}_{2} \mathrm{CH}=\mathrm{CH}_{2}\right), 2.63\left(1 \mathrm{H}\right.$, unresolved br. s, $\left.\mathrm{CH}_{\mathrm{A}} \mathrm{H}_{\mathrm{B}} \mathrm{CH}=\mathrm{CH}_{2}\right), 2.15-2.10(1 \mathrm{H}, \mathrm{m}$, $\left.\mathrm{CH}_{\mathrm{A}} \mathrm{H}_{\mathrm{B}} \mathrm{CH}=\mathrm{CH}_{2}\right), 2.05-1.85\left(2 \mathrm{H}, \mathrm{m}, \mathrm{CH}_{2} \mathrm{CH}_{2}\right), 1.76-1.62\left(2 \mathrm{H}, \mathrm{m}, \mathrm{CH}_{2} \mathrm{CH}_{2}\right), 1.45$ (9 $\left.\mathrm{H}, \mathrm{s}, \mathrm{CMe}_{3}\right) ;{ }^{13} \mathrm{C} \mathrm{NMR}\left(100 \mathrm{MHz}, \mathrm{CDCl}_{3}, 25^{\circ} \mathrm{C}\right) \delta=155.0,140.1,135.4,116.8$, 114.0, 79.3, 60.6, 58.3, 39.5, 30.9, 28.5, 28.0; MS m/z (EI) 196 ( $\mathrm{M}^{+}$-allyl, 12\%), 140 ( $\mathrm{M}^{+}$-allyl- ${ }^{t} \mathrm{Bu}, 50 \%$ ), 57 (100\%); HRMS Found: $m / z$ (EI) $196.1340 \quad \mathrm{C}_{11} \mathrm{H}_{19} \mathrm{O}_{2} \mathrm{~N}$ requires 196.1338; Anal. Calcd for $\mathrm{C}_{14} \mathrm{H}_{23} \mathrm{NO}_{2}$ : C, 70.85; H, 9.77; N, 5.90. Found: C, 71.16; H, 9.88; N, 6.00 . 


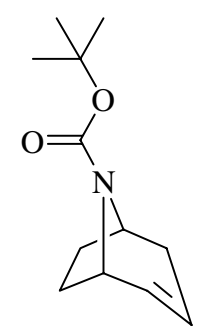

11

To a stirred solution of $\mathbf{1 0}$ (42 mg, $0.18 \mathrm{mmol}, 0.005 \mathrm{M}$ in benzene), was added 1,3(bis(mesityl)-2-imidazolidinylidene)dichloro-(phenylmethylene)(tricyclohexylphosphine) ruthenium II (15.3 $\mathrm{mg}, 0.018 \mathrm{mmol})$. The solution turned yellow upon initial heating, then purple as the mixture was refluxed over 1.5 hours followed by cooling to r.t.. Concentration in vacuo provided a black oil. Purification by column chromatography, eluting with 20\% EtOAc in pet. ether yielded 11 (36.5 mg, 87\%, traces of coloured catalyst impurities) as a slightly impure pale brown oil;

TLC $\mathrm{R}_{f} 0.53$ (20\% EtOAc in pet. ether); $[\alpha]^{20}{ }_{\mathrm{D}}-2.2\left(c\right.$ 0.2, $\left.\mathrm{CHCl}_{3}\right)\{$ lit. +3.0 (c 1.0, $\left.\left.\mathrm{CHCl}_{3}\right)^{[6]}\right\} ; \mathrm{IR} v_{\max }$ (neat) $/ \mathrm{cm}^{-1} 2974,1691,1635 ;{ }^{1} \mathrm{H}$ NMR $\left(400 \mathrm{MHz}, \mathrm{CDCl}_{3}, 25\right.$ ${ }^{\circ} \mathrm{C}$, TMS $) \delta=5.99(1 \mathrm{H}$, unresolved br. s, $\mathrm{NCHCH}=\mathrm{CH}), 5.55-5.49(1 \mathrm{H}, \mathrm{m}$, $\mathrm{NCHCH}=\mathrm{CH}), 4.40-4.18(2 \mathrm{H}, \mathrm{m}, \mathrm{NCH}$ and $\mathrm{NCH}), 2.85-2.65(1 \mathrm{H}, \mathrm{m}$, $\left.\mathrm{CH}=\mathrm{CHCH}_{\mathrm{A}} \mathrm{H}_{\mathrm{B}}\right), 2.22-2.10\left(1 \mathrm{H}, \mathrm{m}, \mathrm{CH}=\mathrm{CHCH}_{\mathrm{A}} H_{\mathrm{B}}\right), 1.99-1.60\left(4 \mathrm{H}, \mathrm{m}, \mathrm{CH}_{2} \mathrm{CH}_{2}\right)$, $1.45\left(9 \mathrm{H}, \mathrm{s}, \mathrm{CMe}_{3}\right) ;{ }^{13} \mathrm{C} \mathrm{NMR}\left(100 \mathrm{MHz}, \mathrm{CDCl}_{3}, 25^{\circ} \mathrm{C}\right) \delta=154.2,133.0,123.6$, $79.2,53.7,34.7,34.0,29.5,28.5,28.4$. 


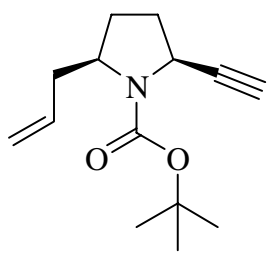

13

To a stirred solution of $\mathrm{CH}_{3} \mathrm{COCN}_{2} \mathrm{PO}(\mathrm{OEt})_{2}(1.14 \mathrm{~g}, 5.92 \mathrm{mmol})$ in $\mathrm{MeOH}(10 \mathrm{ml})$ at r.t. was added $\mathrm{K}_{2} \mathrm{CO}_{3}(1.37 \mathrm{~g}$, $9.88 \mathrm{mmol})$ resulting in a pale yellow solution. After stirring for 10 minutes, a solution of $\mathbf{1 2}(1.18 \mathrm{~g}, 4.94 \mathrm{mmol})$ in $\mathrm{MeOH}(5 \mathrm{ml})$ was added to the reaction mixture, and stirred for 1.5 hours. $\mathrm{H}_{2} \mathrm{O}(15 \mathrm{ml})$ was added and the mixture was extracted with $\mathrm{Et}_{2} \mathrm{O}(3 \times 15 \mathrm{ml})$, the organics were washed with brine $(10 \mathrm{ml})$, dried over $\mathrm{Na}_{2} \mathrm{SO}_{4}$, filtered and concentrated in vacuo to give a clear oil. Purification by column chromatography, eluting with $30 \% \mathrm{Et}_{2} \mathrm{O}$ in pet. ether yielded pure $13(1.08 \mathrm{~g}, 93 \%)$ as a clear oil and as a single diastereomer;

TLC $\mathrm{R}_{f} 0.46$ (30\% $\mathrm{Et}_{2} \mathrm{O}$ in pet. ether); $[\alpha]^{20}{ }_{\mathrm{D}}-30.5$ (c $\left.0.2, \mathrm{CH}_{2} \mathrm{Cl}_{2}\right) ; \mathrm{IR} v_{\max }$ (neat) / $\mathrm{cm}^{-1} 3251,2976,1690,1641 ;{ }^{1} \mathrm{H}$ NMR (400 MHz, $\mathrm{CDCl}_{3}, 25{ }^{\circ} \mathrm{C}$, TMS) $\delta=5.79(1$ $\mathrm{H}$, ddt, $J$ 17, 10 and $\left.7 \mathrm{~Hz}, \mathrm{CH}_{2} \mathrm{CH}=\mathrm{CH}_{2}\right), 5.12-5.03\left(2 \mathrm{H}, \mathrm{m}, \mathrm{CH}_{2} \mathrm{CH}=\mathrm{CH}_{2}\right), 4.51(1$ $\mathrm{H}$, unresolved br. $\mathrm{s}, \mathrm{NCHC} \equiv \mathrm{CH}), 3.83\left(1 \mathrm{H}\right.$, unresolved br. $\mathrm{s}, \mathrm{NCHCH} \mathrm{CH}_{2}=\mathrm{CH}_{2}$ ), $2.64\left(1 \mathrm{H}\right.$, unresolved br. s, $\left.\mathrm{CH}_{\mathrm{A}} \mathrm{H}_{\mathrm{B}} \mathrm{CH}=\mathrm{CH}_{2}\right), 2.31-2.21\left(2 \mathrm{H}, \mathrm{m}, \mathrm{CH}_{\mathrm{A}} \mathrm{H}_{\mathrm{B}} \mathrm{CH}=\mathrm{CH}_{2}\right.$ and $\mathrm{C} \equiv \mathrm{CH}), 2.11-1.81\left(4 \mathrm{H}, \mathrm{m}, \mathrm{CH}_{2} \mathrm{CH}_{2}\right), 1.50\left(9 \mathrm{H}, \mathrm{s}, \mathrm{CMe}_{3}\right) ;{ }^{13} \mathrm{C} \mathrm{NMR}(100 \mathrm{MHz}$, $\left.\mathrm{CDCl}_{3}, 25^{\circ} \mathrm{C}\right) \delta=154.1,135.0,117.1,85.1,79.9,69.9,57.8,48.7,32.0,28.5,28.2$; MS m/z (CI) $180\left(\mathrm{MH}_{2}{ }^{+}-{ }^{t} \mathrm{Bu}, 72 \%\right), 138\left(\mathrm{MH}^{+}\right.$-allyl- $\left.{ }^{t} \mathrm{Bu}, 61 \%\right), 57$ (100\%); HRMS Found: $m / z$ (CI) $180.1023 \mathrm{C}_{10} \mathrm{H}_{14} \mathrm{O}_{2} \mathrm{~N}$ requires 180.1024; Anal. Calcd for $\mathrm{C}_{14} \mathrm{H}_{21} \mathrm{NO}_{2}$ : C, 71.46; H, 8.99; N, 5.95. Found: C, 71.25; H, 8.78; N, 6.03. 


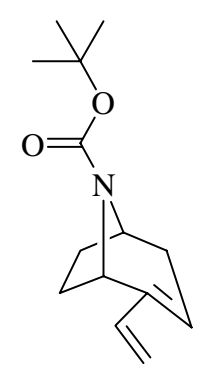

15

To a stirred solution of $\mathbf{1 3}\left(70 \mathrm{mg}, 0.298 \mathrm{mmol}, 0.01 \mathrm{M}\right.$ in $\left.\mathrm{CH}_{2} \mathrm{Cl}_{2}\right)$ was added bis(tricyclohexylphosphine)benzylidine ruthenium (IV) dichloride I (24.5 mg, 0.03 mmol). The solution turned yellow upon initial heating, then purple as the mixture was refluxed over 10 hours followed by cooling to r.t.. Concentration in vacuo provided a black oil. Purification by column chromatography, eluting with $20 \% \mathrm{Et}_{2} \mathrm{O}$ in pet. ether yielded 15 (60.2 $\mathrm{mg}, 86 \%$, traces of coloured catalyst impurities) as a slightly impure brown oil;

TLC $\mathrm{R}_{f} 0.38$ (30\% $\mathrm{Et}_{2} \mathrm{O}$ in pet. ether); $[\alpha]^{20} \mathrm{D}+132.6\left(c 0.2, \mathrm{CH}_{2} \mathrm{Cl}_{2}\right) ; \mathrm{IR} v_{\max }$ (neat) / $\mathrm{cm}^{-1} 1695,1635 ;{ }^{1} \mathrm{H}$ NMR $\left(400 \mathrm{MHz}, \mathrm{CDCl}_{3}, 25^{\circ} \mathrm{C}\right.$, TMS) $\delta=6.22(1 \mathrm{H}, \mathrm{dd}, J 17.5$ and $\left.11 \mathrm{~Hz}, \mathrm{CH}_{2}=\mathrm{CH}\right), 5.45\left(1 \mathrm{H}\right.$, unresolved br. s, $\left.\mathrm{C}=\mathrm{CHCH}_{2}\right), 5.23-5.10(1 \mathrm{H}, \mathrm{m}$, $\left.\mathrm{CH}_{\mathrm{A}} \mathrm{H}_{\mathrm{B}}=\mathrm{CH}\right), 4.97\left(1 \mathrm{H}, \mathrm{d}, J 11.0 \mathrm{~Hz}, \mathrm{CH}_{\mathrm{A}} H_{\mathrm{B}}=\mathrm{CH}\right), 4.78-4.60(1 \mathrm{H}, \mathrm{m}, \mathrm{NCHC}=\mathrm{CH})$, 4.40-4.23 (1 H, m, $\left.\mathrm{NCHCH}_{2} \mathrm{CH}=\mathrm{C}\right), 2.20-2.11$ ( $\left.1 \mathrm{H}, \mathrm{m}, \mathrm{NCHCH}_{\mathrm{A}} \mathrm{CH}_{\mathrm{B}} \mathrm{CH}=\mathrm{C}\right), 2.10$ $1.99\left(1 \mathrm{H}, \mathrm{m}, \mathrm{NCHCH}_{\mathrm{A}} \mathrm{CH}_{\mathrm{B}} \mathrm{CH}=\mathrm{C}\right), 1.94-1.83\left(4 \mathrm{H}, \mathrm{m}, \mathrm{CHCH}_{2} \mathrm{CH}_{2} \mathrm{CH}\right), 1.44(9 \mathrm{H}, \mathrm{s}$, $\left.\mathrm{CMe}_{3}\right) ;{ }^{13} \mathrm{C} \mathrm{NMR}\left(100 \mathrm{MHz}, \mathrm{CDCl}_{3}, 25{ }^{\circ} \mathrm{C}\right) \delta=161.1,142.7,135.9,124.0,110.9$, 79.2, 52.8, 52.5, 34.0, 30.1, 29.7, 28.4; MS m/z (CI) 281 (100\%), $180\left(\mathrm{MH}_{2}{ }^{+}{ }^{t} \mathrm{Bu}\right.$, $25 \%$ ); HRMS Found: $m / z(C I) 180.1020 \mathrm{C}_{10} \mathrm{H}_{14} \mathrm{O}_{2} \mathrm{~N}$ requires 180.1024 . 


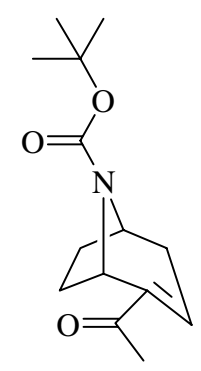

16

To a stirred solution of 15 (37.0 mg, $0.16 \mathrm{mmol})$ in $\mathrm{DMF}(2.5 \mathrm{ml})$ and $\mathrm{H}_{2} \mathrm{O}(1 \mathrm{ml})$ was added $\mathrm{PdCl}_{2}(2.8 \mathrm{mg}$. $0.016 \mathrm{mmol})$ and $\mathrm{CuCl}_{2}(33 \mathrm{mg}, 0.34 \mathrm{mmol})$. The dark green solution was refluxed for 6 hours then cooled to r.t.. $\mathrm{H}_{2} \mathrm{O}(10 \mathrm{ml})$ was added, and the mixture was extracted with $\mathrm{Et}_{2} \mathrm{O}(3 \mathrm{x} 15 \mathrm{ml})$, the organics were washed with $\mathrm{H}_{2} \mathrm{O}(3 \mathrm{x}$ $15 \mathrm{ml})$, brine $(10 \mathrm{ml})$, dried over $\mathrm{Na}_{2} \mathrm{SO}_{4}$, filtered and concentrated in vacuo to give a clear oil. Purification by column chromatography, eluting with $20 \% \mathrm{Et}_{2} \mathrm{O}$ in pet. ether yielded $16(32.5 \mathrm{mg}, 81 \%)$ as a white crystalline solid;

TLC $\mathrm{R}_{f} 0.2$ (30\% $\mathrm{Et}_{2} \mathrm{O}$ in pet. ether); m.p. $61-62{ }^{\circ} \mathrm{C}\left\{\right.$ lit. $\left.^{[7]} 64-65^{\circ} \mathrm{C}\right\}[\alpha]^{20}{ }_{\mathrm{D}}+122.2(c$ $\left.0.2, \mathrm{CHCl}_{3}\right)\left\{\right.$ lit. $^{[7]}+129.1\left(\right.$ c $\left.\left.1.00, \mathrm{CHCl}_{3}\right)\right\} ; \mathrm{IR} v_{\max }$ (neat) $/ \mathrm{cm}^{-1} 1690,1666 ;{ }^{1} \mathrm{H}$ NMR (400 MHz, $\left.\mathrm{CDCl}_{3}, 25^{\circ} \mathrm{C}, \mathrm{TMS}\right) \delta=6.80\left(1 \mathrm{H}, \mathrm{m}, \mathrm{CH}_{2} \mathrm{CH}=\mathrm{C}\right), 4.95(1 \mathrm{H}, \mathrm{m}$, $\mathrm{NCHC}=\mathrm{CH}), 4.32\left(1 \mathrm{H}, \mathrm{m}, \mathrm{NCHCH}_{2} \mathrm{CH}=\mathrm{C}\right), 2.90\left(1 \mathrm{H}, \mathrm{m}, \mathrm{CH}_{\mathrm{A}} \mathrm{H}_{\mathrm{B}} \mathrm{CH}=\mathrm{C}\right), 2.27(3$ $\mathrm{H}, \mathrm{s}, \mathrm{C}=\mathrm{OMe}), 2.17\left(1 \mathrm{H}, \mathrm{m}, \mathrm{CH}_{\mathrm{A}} H_{\mathrm{B}} \mathrm{CH}=\mathrm{C}\right), 2.05(2 \mathrm{H}, \mathrm{m}), 1.86(1 \mathrm{H}, \mathrm{m}), 1.58(1 \mathrm{H}$, m), $1.43\left(9 \mathrm{H}, \mathrm{s}, \mathrm{CMe}_{3}\right)\left\{\right.$ lit. $^{[7]} 6.66(1 \mathrm{H}, \mathrm{m}), 4.92(1 \mathrm{H}, \mathrm{d}, J 5.7 \mathrm{~Hz}), 4.36(1 \mathrm{H}, \mathrm{m})$, $2.93(1 \mathrm{H}, \mathrm{m}), 2.27(3 \mathrm{H}, \mathrm{s}), 2.15(1 \mathrm{H}, \mathrm{m}), 2.04(2 \mathrm{H}, \mathrm{m}), 1.79(1 \mathrm{H}, \mathrm{dt}, J 11.8$ and 2.4 $\mathrm{Hz}), 1.55(1 \mathrm{H}, \mathrm{m}), 1.43(9 \mathrm{H}, \mathrm{s})\} ;{ }^{13} \mathrm{C} \mathrm{NMR}\left(100 \mathrm{MHz}, \mathrm{CDCl}_{3}, 25{ }^{\circ} \mathrm{C}\right) \delta=196.9$, 154.3, 146.2, 137.6, 80.1, 52.6, 51.8, 35.4, 35.0 30.3, 28.9, 25.2 \{ lit. $^{[7]} 196.4,153.9$, 145.5, 137.1, 79.5, 52.0, 51.2, 34.9, 34.6, 29.7, 28.3, 24.8\}; MS m/z (CI) $209\left(\mathrm{MH}^{+}-\right.$ $\left.\mathrm{C}_{2} \mathrm{H}_{3} \mathrm{O}, 12 \%\right) 195\left(\mathrm{MH}^{+}-{ }^{t} \mathrm{Bu}, 35 \%\right), 152\left(\mathrm{MH}^{+}-\mathrm{BOC}, 66 \%\right)$. 
[1] a) Gilbert, J. C.; Weerasooriya, U. J. Org. Chem. 1982, 47, 1837-1845; b) Ohira, S. Synth. Commun. 1989, 19, 561-564; c) Müller, S.; Liepold, B.; Roth, G. J.; Bestmann, H. J. Synlett 1996, 521-522.

[2] Pangborn, A. B.; Giardello, M. A.; Grubbs, R. H.; Rosen, R. K.; Timmers, F. J. Organometallics 1996, 15, 1518-1520.

[3] Gosselin, F.; Lubell, W. D. J. Org. Chem. 2000, 65, 2163-2171.

[4] Van Betsbrugge, J.; Van Den Nest, W.; Verheyden, P.; Tourwe, D. Tetrahedron 1998, 54, 1753.

[5] August, R. A.; Khan, J. A.; Moody, C. M.; Young, D. W. J. Chem. Soc.; Perkin Trans 1, 1996, 507.

[6] Lin, R. H.; Castells, J.; Rapoport, H. J. Org. Chem. 1998, 63, 4069-4078.

[7] Hernández, A. S.; Thaler, A.; Castells, J.; Rapoport, H. J. Org. Chem. 1996, 61, 314-323. 